\title{
Dark Energy Present and Future
}

\author{
Paul H. Frampton \\ Department of Physics and Astronomy, University of North Carolina, Chapel Hill, NC
}

27599-3255, USA

\begin{abstract}
By studying the present cosmological data, particularly on CMB, SNeIA and LSS, we find that the future fate of the universe, for simple linear models of the dark energy equation-ofstate, can vary between the extremes of (I) a divergence of the scale factor in as little as 7 Gyr; (II) an infinite lifetime of the universe with dark energy dominant for all future time; (III) a disappearing dark energy where the universe asymptotes as $t \rightarrow \infty$ to $a(t) \sim t^{2 / 3}$ i.e. matter domination. Precision cosmological data hint that a dark energy with equation of state $w=P / \rho<-1$ and hence dubious stability is viable. Here we discuss for any $w$ nucleation from $\Lambda>0$ to $\Lambda=0$ in a first-order phase transition. The critical radius is argued to be at least of galactic size and the corresponding nucleation rate glacial, thus underwriting the dark energy's stability and rendering remote any microscopic effect.
\end{abstract}

\section{INTRODUCTION}

In this talk I will focus on two issues concerning the enigmatic dark energy which comprises approximately $70 \%$ of the cosmological energy density in the present era.

The first is concerning the future fate of the dark energy and how it depends on the equation of state.

The second is the issue of stability of the dark energy assuming that it can undergo a first-order phase transition to a vacuum of truly zero energy density.

\section{FATE OF DARK ENERGY}

The cosmic concordance of data from three disparate sources: Cosmic Microwave Background (CMB), Large Scale Structure (LSS) and High-Red-Shift Supernovae (SNeIA) suggests that the present values of the dark energy and matter components, in terms of the critical density, are approximately $\Omega_{X} \simeq 0.7$ and $\Omega_{M} \simeq 0.3$. The question to which we try to make a small contribution in this paper is to what extent precision cosmological data will allow us to discriminate between possible futute fates of the Universe?

If one makes the most conservative assumption that $\Omega_{X}$ corresponds to a cosmological constant with Equation of State given by a constant $w=p / \rho=-1$, then the future evolution of the universe follows from the Friedmann equation 


$$
\left(\frac{\dot{a}}{a}\right)^{2}=\frac{8 \pi G \rho}{3}+\frac{\Lambda}{3}
$$

in which $a(t)$ is the scale factor normalized at the present time as $a\left(t_{0}\right)=1, \rho(t)=$ $\rho(0) a^{-3}$ is the energy density of matter component and $\Lambda$ is constant. Here we assumed that the universe is flat and neglected radiation.

In such a simple, and still viable, case the behavior of $a(t)$ for asymptotically large $t \rightarrow \infty$ is

$$
a(t) \sim \exp \left(\sqrt{\frac{\Lambda}{3}} t\right)
$$

so that the dark energy asymptotically dominates and the universe is blown apart in an infinite time $a(t) \rightarrow \infty$ as $t \rightarrow \infty$.

Even assuming that $w$ is constant, however, there is a wide range of possible $w$ : according to [1] the allowed values are $-2.68<w<-0.78$. We do not assume this result but will arrive at a similar allowed range; the difference is because our priors are slightly different (we fix the cosmological parameters $\Omega_{M}=0.3, \Omega_{\Lambda}=0.7, \Omega_{b}=0.02$, and $h=0.65$ instead of allowing them to vary.) The asymptotic behavior in Eq.(2) is very far from established by present data. The case $w<-1$ has the property that boosting from the dark energy rest frame to an inertial frame with velocity satisfying $(v / c)^{2}>-1 / w$ leads to a negative energy density, but this does not violate any law of physics ${ }^{1}$. Here we shall consider some simple models for $w$, including dependence on red-shift $w(z)$, to illustrate how far existing data are from answering the question of the future fate of the universe. As we will show, for a model in which $w$ varies linearly with red-shift, present data are consistent with extremely different futures. For examples, in one case the scale factor diverges[4, 5] in finite time ${ }^{2}$, in just another 7 Gyr, while in another case the energy density of dark energy decreases eventually faster than that of matter, i.e., the dark energy disappears and the universe reverts to being matter-dominated with $a(t) \sim t^{2 / 3}$, as $t \rightarrow \infty$.

\section{Constant Equation of State.}

Here we discuss the future fate of the universe in the case of the constant equation of state. If we assume, to begin, that $w$ is constant then, keeping only the dark energy term

$$
\left(\frac{\dot{a}}{a}\right)^{2}=H_{0}^{2} \Omega_{X} a^{-\beta}
$$

where $\beta=3(1+w)$. Most authors have discussed the case with $w \geq-1$, however the case with $w<-1$ is also possible and discussed phenomenologically in $[1,4,6]$, and in

\footnotetext{
1 This violates the weak energy condition[2, 3].

${ }^{2}$ Gravitationally-bound systems could survive longer than $t_{r}$ in Eq.(5) but such systems would be infinitely separated from one another.
} 
connection with string theory in [7]. If $\beta<0$, corresponding to $w<-1$, the solution of Eq.(3) diverges at a finite time $t=t^{*}$. By integrating

$$
\int_{a\left(t_{0}\right)}^{\infty} a^{\beta / 2-1}=H_{0} \sqrt{\Omega_{X}} \int_{t_{0}}^{t^{*}} d t
$$

one finds that the remaining time $t_{\mathrm{r}}$ before time ends $t_{\mathrm{r}}=\left(t^{*}-t_{0}\right)$ is given analytically by

$$
t_{\mathrm{r}}=\frac{2}{3 H_{0}} \frac{1}{\sqrt{\Omega_{X}}(-w-1)}
$$

In Eq.(5), putting in $\Omega_{X}=0.7$ and $\frac{2}{3} H_{0}^{-1}=9.2$ Gyr one finds for $w=-1.5,-2.0$ and -2.5 , respectively $t_{\mathrm{r}}=22,11$ and $7.3 \mathrm{Gyr}$.

In such a constant $w$ scenario which is consistent with all cosmological data, the divergence of the scale factor will occur in a finite time period of $7 \mathrm{Gyr}$ (or more) from now.

With respect to the Solar System, this end of time occurs generally after the Sun has transformed into a Red Giant, and swallowed the Earth, as is expected approximately 5 Gyr in the future.

Equation of State Varying Linearly with Red-Shift.

To view the four Figures cited, see [25]

As a more general ansatz, we consider the model for $w$ depending linearly on redshift: ${ }^{3}$

$$
w(Z)=w(0)+C Z \theta(\zeta-Z)+C \zeta \theta(Z-\zeta)
$$

where the modification is cut off arbitrarily at some $Z=\zeta>0$. We assume $C \leq 0$ and consider the two-dimensional parameter space spanned by the two variables $w(0)$ and C.

In order to discuss constraints on our phenomenological model, we compare its prediction with experimental data from SNeIA and CMB. We evaluate the goodness-offit parameter $\chi^{2}$ as a function of $w(0)$ and $C$. For SNe1A, we used a dataset consisting of $37 \mathrm{SNe}$ from [10] with the MLCS method. To calculate the CMB power spectrum, we used a modified version of CMBFAST [11]. For the analysis of CMB, we used experimental data from COBE [12], BOOMERanG [13], MAXIMA [14] and DASI [15]. To calculate $\chi^{2}$, we adopt the offset log-normal approximation [16] and used RADPACK package [17]. We also studied the constraint from LSS using the 2dF data [18], and found that it does not give severe constraint on the parameters $w(0)$ and $C$.

\footnotetext{
3 A model with $w$ linearly depending on red-shift is also discussed in [8] but fitting the CMB data was not investigated. Another parametrization of $w(Z)$ is in [9].
} 
For an illustration, we take the cosmological parameters as $\Omega_{\mathrm{b}} h^{2}=0.02, \Omega_{\mathrm{M}}=$ $0.3, \Omega_{\mathrm{X}}=0.7$ and $h=0.65$, and the initial power spectra are assumed to be scale invariant in all numerical calculation in this paper.

To set the stage, let us first use only the SNe1A data to constrain the parameters $w(0)$ and $C$. The result is shown for $\zeta=2$ in Figure 1 where the $99 \% \mathrm{C}$. L. allowed region is the region between the two dashed lines shown. We may remark three distinct regions: (I) $w(0)<(C-1)$. In this case there is divergence of the scale factor, at a finite future time.

(II) $(C-1) \leq w(0)<C$. Here the lifetime of the universe is infinite. The dark energy dominates over matter, as now, at all future times.

(III) $C \leq w(0)$. The lifetime of the universe is again infinite but after a finite time the dark energy will disappear relative to the dark matter and matter-domination will be re-established with $a(t) \sim t^{2 / 3}$.

When we add the constraints imposed by the CMB data, the allowed region is smaller as shown in Figure 2, plotted for $\zeta=0.5$. Such a small $\zeta$ still allows all three future possibilities (I), (II) and (III). For somewhat larger $\zeta$ only possibilities (I) and (II) are allowed in this particular parameterization.

The case $\zeta=2$ is exhibited in more detail for different values of $w(0)$ and $C$ in Figures 3 and 4 . Figure 3 shows the variation of the transition red-shift $Z_{\text {tr }}$ where deceleration changes to accelerated cosmic expansion defined by $q\left(Z_{\mathrm{tr}}\right)=0$. From the figure, we can read off that $Z_{\text {tr }}$ becomes smaller as $C$ becomes more negative for fixed $w(0)$; this is because the epoch where dark energy becomes the dominant component of the Universe becomes later. This affects the magnitude-red shift relation of high- $Z$ supernovae. In Figure 4, the magnitude-red shift relation is shown for the SNeIA data [10] along with the prediction of our phenomenological model for $\zeta=2$. The magnitudes are calculated, as usual, relative to the empty universe Milne model with $\Omega_{M}=0, \Omega_{X}=0$ and $\Omega_{k}=1$. One can expect that high-redshift SNe would appear dimmer if $C$ were more negative.

To return to our main point, let us assume that more precise cosmological data will allow an approximate determination of $w(Z)=f(Z)$ as a function of $Z$ for positive $Z>0$. Then to illustrate the possible future evolutions write:

$$
w(Z)=f(Z) \theta(Z)+(f(0)+\alpha Z) \theta(-Z)
$$

In this case, the future scenarios (I), (II) and (III) occur respectively for $\alpha>(f(0)+1)$, $(f(0)+1)>\alpha>f(0)$ and $\alpha<f(0)$.

Present data are consistent with a simple cosmological constant $f(Z)=-1$ in Eq.(7) in which case the divergence of the scale factor occurs for $\alpha>0$, the infinite-time dark energy domination for $0>\alpha>-1$, and disappearing dark energy for $\alpha<-1$.

Since in practice $F(Z)$ for $Z \geq 0$ will never be determined with perfect accuracy the continuation of $w(Z)$ to future $Z<0$ will be undecidable from observation as will therefore be the ultimate fate of the Universe. 


\section{STABILITY ISSUES FOR DARK ENERGY}

The equation of state for the dark energy component in cosmology has been the subject of much recent discussion [7, 19, 20, 21, 22, 23, 24, 25, 26] Present data are consistent with a constant $w(Z)=-1$ corresponding to a cosmological constant. But the data allow a present value for $w(Z=0)$ in the range $-1.38<w(Z=0)<-0.82$ [19]. If one assumes, more generally, that $w(Z)$ depends on $Z$ then the allowed range for $w(Z=0)$ is approximately the same[25]. In the present article we shall forgo this greater generality as not relevant. Instead, in the present article we address the question of stability for a dark energy with constant $w(Z)<-1$.

\section{Interpretation as a limiting velocity}

Consider making a Lorentz boost along the 1-direction with velocity $V$ (put $\mathrm{c}=1$ ). Then the stress-energy tensor which in the dark energy rest frame has the form:

$$
T_{\mu \nu}=\Lambda\left(\begin{array}{cccc}
1 & 0 & 0 & 0 \\
0 & w & 0 & 0 \\
0 & 0 & w & 0 \\
0 & 0 & 0 & w
\end{array}\right)
$$

is boosted to $T_{\mu \nu}^{\prime}$ given by

$$
T_{\mu \nu}^{\prime}=\Lambda\left(\begin{array}{cccc}
1 & V & 0 & 0 \\
V & 1 & 0 & 0 \\
0 & 0 & 1 & 0 \\
0 & 0 & 0 & 1
\end{array}\right)\left(\begin{array}{cccc}
1 & 0 & 0 & 0 \\
0 & w & 0 & 0 \\
0 & 0 & w & 0 \\
0 & 0 & 0 & w
\end{array}\right)\left(\begin{array}{cccc}
1 & V & 0 & 0 \\
V & 1 & 0 & 0 \\
0 & 0 & 1 & 0 \\
0 & 0 & 0 & 1
\end{array}\right)=\Lambda\left(\begin{array}{cccc}
1+V^{2} w & V(1+w) & 0 & 0 \\
V(1+w) & V^{2}+w & 0 & 0 \\
0 & 0 & w & 0 \\
0 & 0 & 0 & w
\end{array}\right)
$$

We learn several things by studying Eq.(9). First, consider the energy component $T_{00}^{\prime}=$ $1+V^{2} w$. Since $V<1$ we see that for $w>-1$ this is positive $T_{00}^{\prime}>0$ and the Weak Energy Condition (WEC) is respected[27]. For $w=-1, T_{00}^{\prime} \rightarrow 0$ as $V \rightarrow 1$ and is still never negative. For $w<-1$, however, we see that $T_{00}^{\prime}<0$ if $V^{2}>-(1 / w)$ and this violates the WEC and is the first sign that the case $w<-1$ must be studied with great care. Looking at the pressure component $T_{11}^{\prime}$ we see the special role of the case $w=-1$ because $w=T_{11}^{\prime} / T_{00}^{\prime}$ remains Lorentz invariant as expected for a cosmological constant. Similarly the off-diagonal components $T_{01}^{\prime}$ remain vanishing only in this case. The main concern is the negativity of $T_{00}^{\prime}<0$ which appears for $V^{2}>-(1 / w)$. One possibility is that it is impossible for $V^{2}>-(1 / w)$. The highest velocities known are those for the highest-energy cosmic rays which are protons with energy $\sim 10^{20} \mathrm{eV}$. These have $\gamma=\left(1-V^{2}\right)^{-1 / 2} \sim 10^{11}$ corresponding to $V \sim 1-10^{-22}$. This would imply that:

$$
w>-1-10^{-22}
$$


which is one possible conclusion.

First-Order Phase Transition and Nucleation Rate.

But let us suppose, as hinted at by $[19,20,21]$ that more precise cosmological data reveals a dark matter which violates Eq.(10). Then, by boosting to an inertial frame with $V^{2}>-(1 / w)$, one arrives at $T_{00}^{\prime}<0$ and this would be a signal for vacuum instability[30]. If the cosmological background is a Friedmann-Robertson-Walker (FRW) metric the physics is Lorentz invariant and so one should be able to see evidence for the instability already in the preferred frame where $T_{\mu \nu}$ is given by Eq.(8). This goes back to work in the 1960's and 1970's where one compares the unstable vacuum to a superheated liquid. As an example, at one atmospheric pressure water can be heated carefully to above $100^{\circ} \mathrm{C}$ without boiling. The superheated water is metastable and attempts to nucleate bubbles containing steam. However, there is an energy balance for a three-dimensional bubble between the positive surface energy $\sim R^{2}$ and the negative latent heat energy of the interior $\sim R^{3}$ which leads to a critical radius below which the bubble shrinks away and above which the bubble expands and precipitates boiling $[28,29]$. For the vacuum the first idea in [30] was to treat the spacetime vacuum as a four-dimensional material medium just like superheated water. The second idea in the same paper was to notice that a hyperspherical bubble expanding at the speed of light is the same to all inertial observers. This Lorentz invariance provided the mathematical relationship between the lifetime for unstable vacuum decay and the critical radius of the four-dimensional bubble or instanton.

In the rest frame, the energy density is

$$
T_{00}=\Lambda \sim\left(10^{-3} e V\right)^{4} \sim(1 \mathrm{~mm})^{-4}
$$

since $10^{-3} \mathrm{eV} \sim(1 \mathrm{~mm})^{-1}$.

In order to make an estimate of the dark energy decay lifetime in the absence of a known potential, we can proceed by assuming (without motivation from observation) that there is a first-order phase transition possible between the $\Lambda=\left(10^{-3} \mathrm{eV}\right)^{4}$ "phase" and $a \Lambda=0$ "phase". This hypothesized decay is the Lorentz invariant process of a hyperspherical bubble expanding at the speed of light, the same for all inertial observers. Let the radius of this hypersphere be $\mathrm{R}$, its energy density be $\varepsilon$ and its surface tension be $S_{1}$. Then according to [30] the relevant instanton action is

$$
A=-\frac{1}{2} \pi^{2} R^{4} \varepsilon+2 \pi^{2} R^{3} S_{1}
$$

where $\varepsilon$ and $S_{1}$ are the volume and surface energy densities, respectively. The stationary value of this action is

$$
A_{m}=\frac{27}{2} \pi^{2} S_{1}^{4} / \varepsilon^{3}
$$

corresponding to the critical radius

$$
R_{m}=3 S_{1} / \varepsilon
$$


We shall assume that the wall thickness is negligible compared to the bubble radius. The number of vacuum nucleations in the past lightcone is estimated as

$$
N=\left(V_{u} \Delta^{4}\right) \exp \left(-A_{m}\right)
$$

where $V_{u}$ is the 4-volume of the past and $\Delta$ is the mass scale relevant to the problem. This vacuum decay picture led to the proposals of inflation[31], for solving the horizon, flatness and monopole problems (only the horizon problem was generally known at the time of [30]). None of that work addressed why the true vacuum has zero energy. Now that the observed vacuum has non-zero energy density $+\varepsilon \sim\left(10^{-3} \mathrm{eV}\right)^{4}$ we may interpret it as a false vacuum lying above the true vacuum with $\varepsilon=0$. In order to use the full power of Eq.(15) taken from [30,32] and the requirement $N \ll 1$ we need to estimate the three mass-dimension parameters $\varepsilon^{1 / 4}, S_{1}^{1 / 3}$ and $\Delta$ therein and so we discuss these three scales in turn.

The easiest of the three to select is $\varepsilon$. If we imagine a tunneling through a barrier between a false vacuum with energy density $\varepsilon$ to a true vacuum at energy density zero then the energy density inside the bubble will be $\varepsilon=\Lambda=\left(10^{-3} \mathrm{eV}\right)^{4}$. No other choice is reasonable. As for the typical mass scale $\Delta$ in the prefactor of Eq. (15), the value of $\Delta$ does not matter very much because it appears in a power rather than an exponential so let us put (the reader can check that the conclusions do not depend on this choice) $\Delta=\varepsilon^{1 / 4}=(1 \mathrm{~mm})^{-1}$ whereupon the prefactor in Eq. $(15)$ is $\sim 10^{116}$. The third and final scale to discuss is the surface tension, $S_{1}$. Here we appeal to comparison of spontaneous decay to stimulated decay. The former dictates $N \ll 1$ in Eq.(15): the latter requires further discussion.

Spontaneous dark energy decay brings us to the question of whether such decay can be initiated in an environment existing within our Universe. The question is analogous to one of electroweak phase transition in high energy particle collision. This was first raised in [30] and revisited for cosmic-ray collisions in [33]. That was in the context of the standard-model Higgs vacuum and the conclusion is that high-energy colliders are safe at all present and planned foreseeable energies because much more severe conditions have already occurred (without disaster) in cosmic-ray collisions within our galaxy. More recently, this issue has been addressed in connection with fears that the Relativistic Heavy Ion Collider (RHIC) might initiate a diastrous transition but according to careful analysis $[34,35]$ there was no such danger.

The energy density involved for dark energy is some 58 orders of magnitude smaller $\left[\left(10^{-3} \mathrm{eV}\right)^{4}\right.$ compared to $\left.(300 \mathrm{GeV})^{4}\right]$ than for the electroweak case and so the nucleation scales are completely different. One is here led away from microscopic towards astronomical size scales.

The energy density of Eq.(11) is so readily exceeded that the critical radius cannot be microsopic. Think first of a macroscopic scale e.g. 1 meter and consider a magnetic field practically-attainable in bulk on Earth such as 10 Tesla. Its energy density is given by

$$
\rho_{\text {mag }}=\frac{1}{\mu_{0}} \mathscr{B}^{2}
$$


Using the value $\mu_{0}=4 \pi \times 10^{-7} \mathrm{NA}^{-2}$ and $1 T=6.2 \times 10^{12}\left(\mathrm{MeV} . \mathrm{s} . \mathrm{m}^{-2}\right)$ leads to an energy density $\rho_{\text {mag }}=2.5 \times 10^{17} \mathrm{eV} /(\mathrm{mm})^{3}$, over 20 orders above the value of Eq.(11) for the interior of nucleation. Magnetic fields in bulk exist in galaxies with strength $\sim 1 \mu G$ and the rescaling by $\mathscr{B}^{2}$ then would give $\rho_{\text {mag }} \sim\left(2.5 \times 10^{-5} \mathrm{eV}\right)(\mathrm{mm})^{-3}$, slightly below the value in Eq.(11).

Assuming the dark energy can exchange energy with magnetic energy density the observed absence of stimulated decay would then imply a critical radius of at least galactic size, say, $\sim 10 \mathrm{kpc}$. Using $\operatorname{Eq}(14)$ then gives for the surface tension $S_{1}>10^{23}(\mathrm{~mm})^{-3}$ and number of nucleations in Eq.(15) $N<\exp \left(-10^{92}\right)$. The spontaneous decay is thus glacial. Note that the dark energy has appeared only recently in cosmological time and has never interacted with background radiation of comparable energy density. Also, this nucleation argument does not require $w<-1$.

\section{DISCUSSION}

As a first remark, since the critical radius $R_{m}$ for nucleation is astronomical, it appears that the instability cannot be triggered by any microscopic process. While it may be comforting to know that the dark energy is not such a doomsday phenomenon, it also implies at the same time the dreadful conclusion that dark energy may have no microscopic effect. If any such microscopic effect in a terrestrial experiment could be found, it would be crucial in investigating the dark energy phenomenon. We note that the present arguments are less model-dependent than those in [26].

In closing one may speculate how such stability arguments may evolve. One may expect most conservatively that the value $w=-1$ will eventually be established empirically in which case both quintessence and the "phantom menace" will be irrelevant. In that case, indeed for any $w$, we may still hope that dark energy will provide the first connection between string theory and the real world as in e.g. [23]. Even if precise data do establish $w<-1$, as in the "phantom menace" scenario, the dark energy stability issue is still under control.

\section{ACKNOWLEDGEMENT}

I thank C.N. Leung, S. Tovey and R. Volkas for organizing this stimulating workshop. This work was supported in part by the Department of Energy under Grant Number DE-FG02-97ER-410236. 


\section{REFERENCES}

1. S. Hannestad and E. Mörtsell. Phys. Rev. D66, 063508 (2002). ast ro-ph / 0205096.

2. S.W. Hawking, Comm. Math. Phys. 25, 152 (1972).

3. L.H. Ford. gr-qC/0301045

4. R.R. Caldwell. Phys. Lett. B545, 23 (2002). astro-ph/9908168.

5. A.A. Starobinsky, Grav. Cos. 6, 157 (2000). astro-ph/ 9912054.

6. A.E. Schulz and M. White, Phys. Rev. 64, 043514 (2001). astro-ph/ 0104112.

P. Schuecker, et al. astro-ph/0211480.

A. Melchiorri, et al. astro-ph/0211522.

7. P.H. Frampton, Phys. Lett. B555, 139 (2003). ast ro-ph/ 0209037. M. Bastero-Gil, P.H. Frampton and L. Mersini, Phys. Rev. D65, 106002 (2002).

8. D.A. Dicus and W.W. Repko, hep-ph/0211109.

9. E.V. Linder. astro-ph/0208512 and astro-ph/0210217.

10. A.G. Riess et al., Astronomical J. 106, 1009 (1998).

11. U. Seljak and M. Zaldarriaga, Astrophys. J. 469, 437 (1996).

12. C. Bennett et al., Astrophys. J. Lett. 464 (1996) L1.

13. C. B. Netterfield et al., Astrophys. J. 571, 604 (2002).

14. A. T. Lee et al., Astrophys. J. Lett. 561, L1 (2002),

15. N. W. Halverson et al., Astrophys. J. 568, 38 (2002),

16. J. R. Bond, A.H. Jaffe and L. Knox, Astrophys. J. 533, 19 (2000).

17. RADPACK webpage, http://flight.uchicago.edu/knox/radpack.html.

18. M. Tegmark, A. J. S. Hamilton and Y. Xu, MNRAS 335, 887 (2002). astro-ph 0111575.

19. A. Melchiorri, L. Mersini, C.J. Odman and M. Trodden. astro-ph/ 0211522.

20. P. Schuecker, R.R. Caldwell, H. Bohringer, C.A. Collins and L. Guzzo. ast ro-ph/ 0211480.

21. S. Hannestad and E. Mörtsell. Phys. Rev. D66, 063508 (2002). astro-ph / 0205096.

22. R.R. Caldwell, Phys. Lett. B545, 23 (2002). astro-ph/9908168. astro-ph/ 0209037.

23. M. Bastero-Gil, P.H. Frampton and L. Mersini, Phys. Rev. D65, 106002 (2002).

24. D.A. Dicus and W.W. Repko. hep-ph/0211109.

25. P.H. Frampton and T. Takahashi, Phys. Lett. B557, 135 (2003). astro-ph / 0211544.

26. S.M. Carroll, M. Hoffman and M. Trodden. astro-ph/0301273

27. S.W. Hawking, Comm. Math. Phys. 25, 152 (1972).

L.H. Ford. gr-qc/0301045.

28. J. Langer, Ann. Phys. 41, 108 (1967).

29. J. Langer, Ann. Phys. 54, 258 (1969).

30. P.H. Frampton, Phys. Rev. Lett. 37, 1378 (1976).

31. A.H. Guth, Phys. Rev. D23, 347 (1981).

A.D. Linde, Phys. Lett. B108, 389 (1982).

A. Albrecht and P.J. Steinhardt, Phys. Rev. Lett. 48, 1220 (1982).

32. P.H. Frampton, Phys. Rev. D15, 2922 (1977).

33. P. Hut, Nucl. Phys. A418, 301C (1984).

P. Hut and M.J. Rees, IAS-Princeton preprint 83-0042 (1984)

34. R.L. Jaffe, W. Busza, F. Wilczek and J. Sandweiss, Rev. Mod. Phys. 72, 1125 (2000).

35. A. Dar, A. De Rujula and U. Heinz, Phys. Lett. B470, 142 (1999). 